\title{
Assessment of transfemoral amputees using C-Leg and Power Knee for ascending and descending inclines and steps
}

\author{
Erik J. Wolf, PhD; ${ }^{1-2^{*}}$ Vanessa Q. Everding, MS; ${ }^{2}$ Alison L. Linberg, DPT; ${ }^{1-2}$ Barri L. Schnall, MPT; ${ }^{2}$ Joseph $^{2}$ \\ M. Czerniecki, MD; ${ }^{3}$ Jeffrey M. Gambel, $\mathrm{MD}^{2}$ \\ ${ }^{1}$ Henry M. Jackson Foundation for the Advancement of Military Medicine, Bethesda, MD; ${ }^{2}$ Department of Ortho- \\ paedics and Rehabilitation, Walter Reed Army Medical Center, Washington, DC; ${ }^{3}$ Department of Veterans Affairs \\ Puget Sound Health Care System, Seattle, WA; and Department of Rehabilitation Medicine, University of Washington, \\ Seattle, WA
}

\begin{abstract}
Adding active power to a prosthetic knee unit may improve function and reduce the potential for overuse injuries in persons with transfemoral amputation (TFA). Servicemembers who have sustained a TFA are often young and motivated to perform at high functional levels. The goal of this article is to compare the biomechanics of ramp and stair descent and ascent for participants using the C-Leg and the Power Knee (PK). Subjects were asked to ascend and descend an instrumented staircase and 12 degree ramp at their comfortable pace while equipped with retroreflective markers. Temporal-spatial and kinetic data were collected. Knee power generated by the nondisabled limb during stair ascent for subjects wearing the C-Leg was significantly greater than for those wearing the PK. Knee power generated by prosthetic knee units was significantly greater for subjects while wearing the PK. Although the PK reduced the power required from the nondisabled knee during stair climbing, it does not appear to be superior to the CLeg for other tasks. Adding power to a prosthetic knee may reduce wear on the nondisabled limb; however, there are still limitations that require improvement.
\end{abstract}

Key words: amputation, ankle, biomechanics, hip, kinetics, knee, power, prosthesis, servicemember, unilateral.

\section{INTRODUCTION}

Over 840 U.S. military servicemembers have sustained a major lower-limb amputation as a result of the current conflicts, Operation Iraqi Freedom/Operation Enduring Freedom (OIF/OEF) [1]. These servicemembers are almost always extremely motivated to reach their highest level of function. Whether remaining on Active Duty or eventually separating to civilian life, each individual will continue to have many physical obstacles to negotiate, including stairs and slopes.

The loss of a limb, and the respective anatomical joints, has been shown to cause a deviation from "normalized" mobility and can result in asymmetries during gait [2-8]. These asymmetries have been reported to contribute to secondary injuries such as osteoarthritis, joint degeneration, and low-back pain [7,9-15]. Servicemembers with trauma, including amputation, to one or both lower limbs may be at a greater risk for secondary injuries because of additional physical compensations during ambulation. Ascending and descending slopes and stairs present a greater biomechanical challenge than overground walking.

Abbreviations: OIF/OEF = Operation Iraqi Freedom/Operation Enduring Freedom, PK = Power Knee, TFA = transfemoral amputation.

*Address all correspondence to Erik J. Wolf, PhD; Walter Reed National Military Medical Center, America Building No. 19, Room B322, 8901 Rockville Pike, Bethesda, MD 20889; 301-400-2082. Email: erik.wolf@med.navy.mil http://dx.doi.org/10.1682/JRRD.2010.12.0234 
The additional difficulties associated with slopes and stairs may translate into additional degeneration of the remaining joints caused by increased forces and moments compared with overground walking.

Research has shown that for young nondisabled individuals, joint forces and moments at the knees and hips are greater during stair ascent than descent or level walking. This implies a greater demand on those joints [1617]. Minimal research has been conducted on ambulation of those with major limb loss during stair and ramp ascent and descent. One study examined stair descent for persons with transfemoral amputation (TFA) and transtibial amputation and stair ascent for those with transtibial amputation only [18]. They concluded that during stair descent, people with TFA respond more similarly to controls than people with transtibial amputation. This result occurs because people with TFA who use a microprocessor knee are better able to control knee hydraulics during the descent than those with mechanical knees. Better knee hydraulics allow a person using a microprocessor knee to control their descent. During ascent, functional compensations are made primarily by the contralateral limb [18]. The compensations and resulting forces on the nondisabled limb may be even greater for those with TFA because of loss of a functional knee on the involved side.

For people who have TFA, stair climbing is a challenging functional task. One study reports that only 7.2 percent of people with TFA are able to ascend stairs without handrail assistance and only 3 percent descend stairs step-over-step [19]. This result differs from previously mentioned research [18], which reports people with TFA descending predominantly step-over-step. These differing results may have occurred because of the prosthetic technologies used for the studies cited and when the studies occurred (1984 vs 2007). These data highlight the difficulty of this task and the importance of identifying solutions that will ease the burden of stair ambulation, particularly for people with TFA. Compared with stair ambulation, ramp ambulation was easier for these individuals because 99 percent required no assistance to ascend or descend ramps while 1 percent needed assistance [19]. This does not imply that sloped gait is not difficult for persons with TFA. One study shows that kinematic adjustment strategies are employed while negotiating slopes [20].

Efforts to replace knee function with mechanical devices have been met with varying degrees of success.
The literature contains an ample number of biomechanical studies that, through the years, can be shown to correlate improved gait dynamics with technological advances in prosthetic knee design [21-23]. Decreased frequency of falls and stumbles and increased user satisfaction have also been demonstrated with the use of microprocessorcontrolled prosthetic knee units compared with mechanical devices [24-25]. The effect of prosthetic knee type on stair ambulation has received very limited study. People with TFA performed significantly better on the Stair Assessment Index when using the microprocessor controlled C-Leg compared with mechanical knees during stair descent, but there was no significant difference in stair ascent [24].

The overall objective of this article is to evaluate the biomechanics of servicemembers with TFA using the Power Knee (PK, Össur Americas; Foothill Ranch, California) and C-Leg (Otto Bock Healthcare; Minneapolis, Minnesota) during stair and slope ascent and descent. We hypothesized that the PK would normalize the biomechanics (result in values closer to those of nondisabled subjects) of the prosthetic limb during stair and ramp ascent while reducing the reliance on adaptations of the nondisabled limb as defined by temporal-spatial parameters (step length and stance percentage), knee power generation, and knee absorption during these tasks. We expected these outcomes because the PK provides powered extension to assist in stair and ramp ascent. In contrast, we expected the biomechanical characteristics of stair and ramp descent to be similar between the two knee types because both prosthetic knee units control knee flexion through microprocessors. Active knee extension, the main difference the PK provides, is not a driving factor in stair descent.

\section{METHODS}

We recruited 5 OIF/OEF servicemembers with unilateral TFA to participate in this study from a cohort of 10 OIF/OEF servicemembers with unilateral TFA who had completed another protocol comparing the C-Leg with the PK. The sample consisted entirely of male subjects. Two participants had amputations involving the left femur; three had amputations involving the right femur. All patients were independent ambulators and reported medium to high daily activity levels. All patients wore the Low Profile Vari-Flex foot (Össur Americas) with both 
prosthetic knee types during testing. The Low Profile Vari-Flex foot is an energy storage and return foot. We selected this prosthetic foot because it is commonly prescribed to our patient population and can be used with both prosthetic knee units. We selected the two prosthetic knee units for testing in this study because at the time of the study's inception, the C-Leg was the most commonly prescribed prosthetic knee unit at Walter Reed Army Medical Center (Washington, DC) and the PK was the first commercially available knee unit to provide active extension. While both the C-Leg and the PK are controlled by microprocessors, they differ in that the C-Leg can only provide resistance through hydraulics. The PK provides resistance as well as active torque of the knee though electric motors.

Inclusion criteria for subjects included a comfortable total surface bearing socket (without locking pin); independence as a community ambulator without an assistive device; and no contralateral limb injuries or comorbidities that significantly affected gait, joint range of motion, or limb muscle activity. We documented comfort, including pain levels, skin health, and overall fit, before testing. Subjects in the original study received 6 weeks of training specific to each of the prosthetic knee units to assure expertise in using the advanced functions of both prostheses. This timeline was established by a physical therapist and prosthetist involved in the study to ensure that the subjects were well trained, comfortable, and safe using both knee units. Subjects had to complete the 6 weeks of training and demonstrate specific expertise in the use of each of the prosthetic knee units, including using all modes of each prosthesis and performing set functional tasks. For this protocol, we asked subjects to perform two tests, once using a C-Leg and again using a PK. We allotted a minimum of 3 weeks between data collections to allow for users to reacclimatize to either the C-Leg or the PK, depending on which knee unit we tested first. The order of testing was not randomized. We gave the subject the option of which knee to use first in the study.

After they consented to participate, we fit subjects with 36 retroreflective markers placed on specific anatomical landmarks of their lower limbs. We used a Cleveland Clinic marker set with thigh and shank plates with four markers secured to identify the knee and ankle joint centers. We collected three-dimensional position data from the markers at $120 \mathrm{~Hz}$ with 10 motion capture cameras (Vicon; Los Angeles, California). We asked subjects to perform four tasks for each testing session: ascending and descending a $12^{\circ}$ ramp $(5.75 \mathrm{~m})$ and ascending and descending a 16 -step staircase (7 in. rise to $11.5 \mathrm{in}$. run). The ramp and staircase were instrumented with two AMTI force platforms (Advanced Mechanical Technology Inc; Watertown, Massachusetts). We collected data from the force platforms at $1,200 \mathrm{~Hz}$. For the stair condition, we mounted stairs to the force platforms so that steps 1 and 3 detected force from platform 1 and steps 2 and 4 detected force from platform 2 .

We asked participants to ascend and descend the ramp at a comfortable speed and instructed them to use the handrails as little as possible. We collected data until five "clean" strikes were recorded for each limb. To be considered a clean trial, the subject had to have either foot completely within the boundary of the force platform. We then asked participants to ascend and descend the staircase at a comfortable speed and progress stepover-step. Again, they were advised to use the handrails as little as possible. Subjects averaged approximately 15 trials to accomplish the ramp conditions and approximately 8 trials to complete the stair conditions. Subjects were allowed rest periods at any point during the testing to minimize fatigue and ensure subject safety.

We reduced raw motion-capture data using Vicon Nexus software (Vicon). We removed erroneous markers and filtered data using a second-order Butterworth filter with a cutoff frequency of $6 \mathrm{~Hz}$ and passed bidirectionally. We calculated kinetic data using a 7-segment biomechanical model developed in Visual3D software (CMotion Inc; Germantown, Maryland). We compared temporal-spatial (speed, stance percentage, and step length) and kinetic (vertical ground reaction force and ankle, knee, and hip powers) data between prosthetic knee units.

We performed a paired $t$-test to determine whether significant differences existed between C-Leg and PK data. Evaluations were made separately for the subjects' nondisabled limb and prosthetic limb. We considered results significant for $p<0.05$. We collected control data as part of this study from five nondisabled servicemembers. We evaluated these data to provide normative information for this population performing stair and slope ambulation.

\section{RESULTS}

Table 1 lists demographics of the sample. Control subjects had a mean \pm standard deviation weight of $85 \pm$ $8 \mathrm{~kg}$ and height of $177 \pm 7 \mathrm{~cm}$. 
Table 1.

Subject demographics.

\begin{tabular}{cccc}
\hline Subject & $\begin{array}{c}\text { Time Since } \\
\text { Amputation } \\
(\mathbf{y r})\end{array}$ & $\begin{array}{c}\text { Height } \\
\mathbf{( c m )}\end{array}$ & $\begin{array}{c}\text { Weight } \\
\mathbf{( k g )}\end{array}$ \\
\hline 1 & 2.4 & 178.0 & 80.6 \\
2 & 1.9 & 175.0 & 66.0 \\
3 & 1.5 & 175.0 & 91.0 \\
4 & 1.5 & 172.0 & 74.4 \\
5 & 5.4 & 184.5 & 76.4 \\
Mean \pm Standard & $2.5 \pm 1.6$ & $176.9 \pm 4.8$ & $77.7 \pm 9.1$ \\
Deviation & & & \\
\hline \hline
\end{tabular}

\section{Descending Ramp and Stairs}

Tables 2 and $\mathbf{3}$ show the temporal-spatial and kinetic results for descending the ramp and stairs for subjects using the C-Leg and the PK. Subjects spent significantly more time on their nondisabled limb (75\% for PK vs $70 \%$ for C-Leg) and less time on their prosthetic limb using the PK (55\% for PK vs 57\% for C-Leg). Additionally, subjects took a longer step with their prosthetic limb while wearing the C-Leg $(0.55 \mathrm{~m}$ for PK vs $0.62 \mathrm{~m}$ for CLeg). There were no significant differences in the temporal-spatial variables during stair descent between the PK and C-Leg. Figures 1 and $\mathbf{2}$ show representative data from single subjects of the ankle, knee, and hip power during ramp and stair descent using the $\mathrm{PK}$ and the CLeg. Data are also displayed for uninjured servicemembers during ramp and stair descent. Participants with TFA showed significantly greater ankle power generation on the nondisabled limb during stair descent while wearing the PK (4.8 W/kg vs $3.8 \mathrm{~W} / \mathrm{kg}$ for C-Leg) and significantly greater late-stance knee power absorption on the prosthetic limb during stair descent while wearing the PK $(-2.9 \mathrm{~W} / \mathrm{kg}$ vs $-2.3 \mathrm{~W} / \mathrm{kg}$ for C-Leg). Late stance is defined as the time between midstance and foot-off.

\section{Ascending Ramp and Stairs}

Tables $\mathbf{4}$ and $\mathbf{5}$ show the temporal-spatial and kinetic results for ascending the ramp and stairs for subjects using either the C-Leg or the PK. During ramp ascent, subjects took a significantly longer step with their prosthetic limb while wearing the C-Leg $(0.52 \mathrm{~m}$ for PK vs $0.61 \mathrm{~m}$ for C-Leg). There were no significant differences in other temporal-spatial variables during ramp and stair ascent. Figures $\mathbf{3}$ and $\mathbf{4}$ show representative data from single subjects of the ankle, knee, and hip power during ramp and stair ascent using the PK and the C-Leg. Data are also displayed for nondisabled controls during ramp and stair ascent. Participants with TFA showed significantly greater early-stance knee power generation on the prosthetic limb during ramp and stair ascent while wearing the PK (1.9 W/kg for PK vs $0.2 \mathrm{~W} / \mathrm{kg}$ for C-Leg). Early stance is defined as the time from heel strike to midstance. Subjects did not show late-stance knee power generation while ascending the ramp. During stair ascent, participants showed significantly greater late-stance knee power generation on the nondisabled limb while wearing the C-Leg $(9.0 \mathrm{~W} / \mathrm{kg}$ vs $2.1 \mathrm{~W} / \mathrm{kg}$ for PK) and on the prosthetic limb while wearing the PK $(1.2 \mathrm{~W} / \mathrm{kg}$ vs $0.3 \mathrm{~W} / \mathrm{kg}$ for C-Leg). Participants with TFA also showed significantly greater nondisabled hip power generation during ramp ascent while using the C-Leg.

\section{DISCUSSION}

Functional and clinically relevant goals of the PK are to provide power to the affected knee and relieve stress to the nondisabled limb. Long-term effects associated with major limb loss include osteoarthritis and related pain of the nondisabled limb [14-15]. The goal of this article was to evaluate servicemembers using the PK and the C-Leg

Table 2.

Temporal-spatial data for descending ramp and stairs.

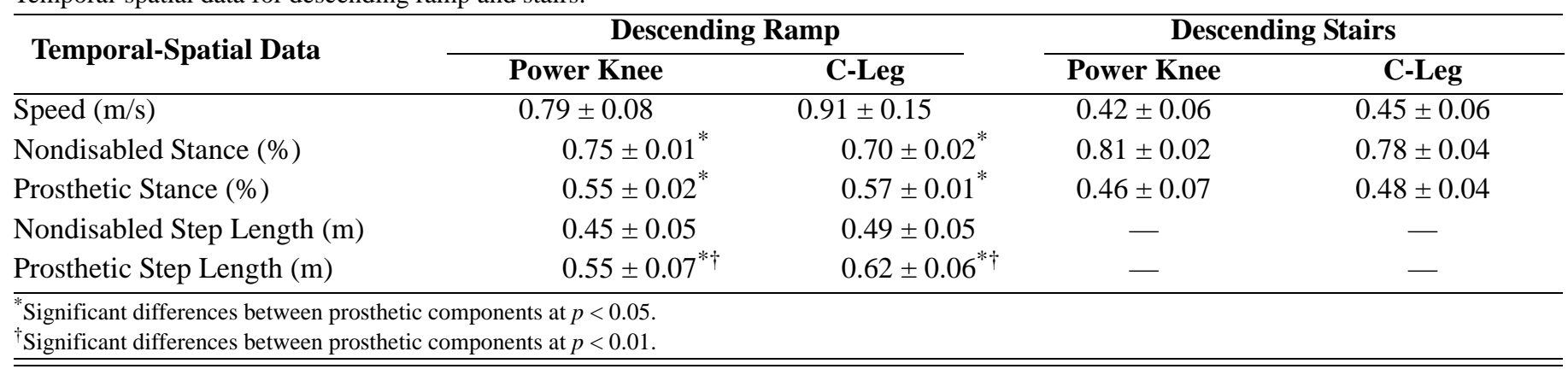


Table 3.

Power data for descending ramp and stairs.

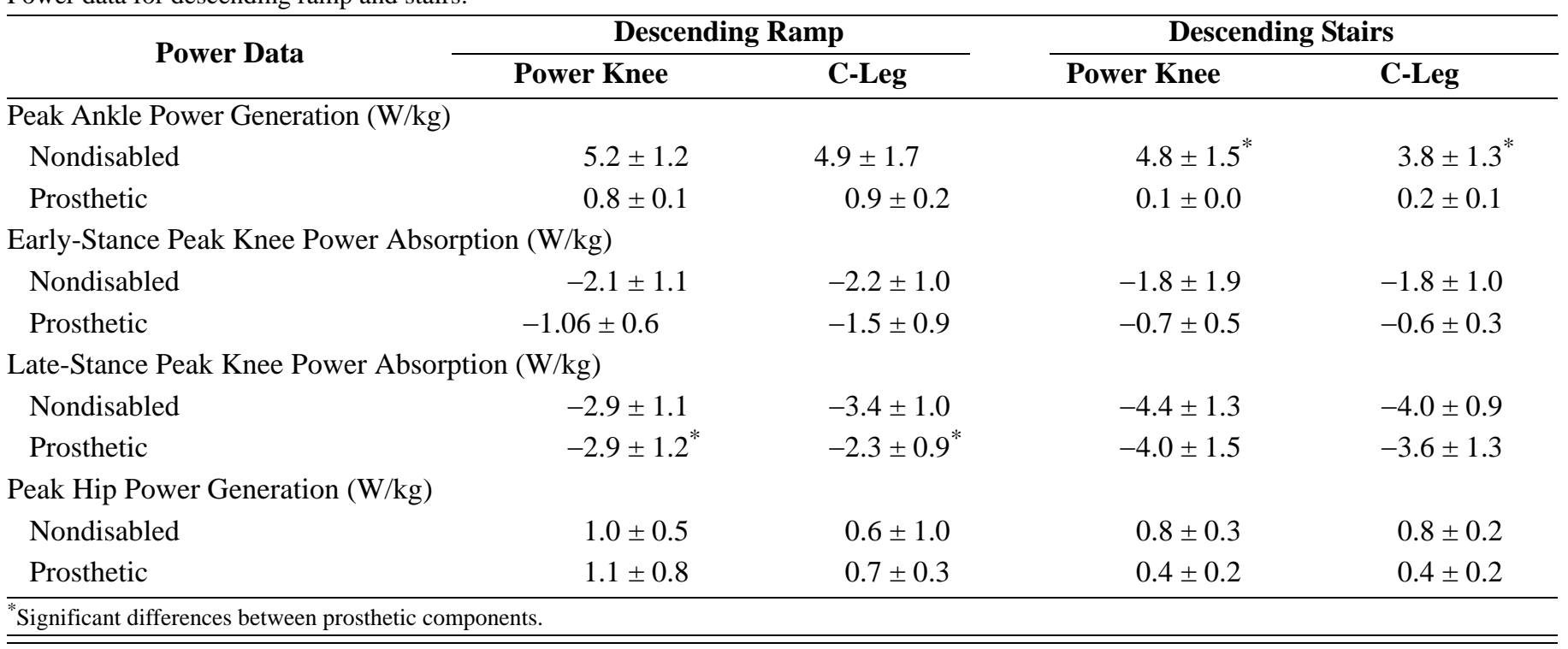

while ascending and descending slopes and stairs to determine whether the active propulsion provided by the PK can significantly improve the biomechanics of the user.

\section{Descending Ramp and Stairs}

Results from subjects descending stairs and ramps showed significant kinetic differences for the C-Leg compared with the PK at the nondisabled ankle while descending stairs and within the temporal-spatial data while descending the ramp. These results are a deviation from the original hypothesis that there would be no differences during descent because both knee units are controlled by microprocessors. During ramp descent, subjects demonstrated longer step lengths bilaterally using the C-Leg, with the prosthetic comparison with the PK showing significance. This is likely because of the increased walking speed of the subjects while wearing the C-Leg. Subjects spent more time in stance on their nondisabled limb and less time on their prosthetic limb during both ramp and stair descent. This result is consistent with the overground walking literature $[4,26]$. Comparison between knee units showed that subjects using the C-Leg spent significantly less time on their nondisabled limb and significantly more time on their prosthetic limb compared with the PK. This asymmetry is similar for stair descent although not significant. This result indicates a greater reliance on the C-Leg compared with the PK during descent. Other researchers reporting on stance time in subjects with TFA have concluded that increased nondisabled stance time implies increased sense of security and comfort [26].

Subjects showed increased ankle power generation at the PK compared with the C-Leg, and as indicated in Figure 2, both conditions appear to be greater than the control group. This result may be due to a strategy to maintain safety during stair descent. The subjects may be keeping their center of mass more posterior while leading with their prosthetic limb to maintain stability and safety. The ankle power generation on the nondisabled limb occurs during double support, after the prosthetic limb has made contact. Once the subject feels secure, additional power from the nondisabled limb may be needed to both overcome the lag of the center of mass and potentially to trigger the prosthetic limb to react with proper functionality associated with stair descent. This phenomenon needs to be examined more closely with future research. Based on these results, subjects may have more confidence in their C-Leg while descending stairs and ramps.

\section{Ascending Ramp and Stairs}

Temporal-spatial results from subjects ascending ramps and stairs only showed significant differences between the C-Leg and the PK for prosthetic step length during ramp ascent. Although not statistically significant, subjects ascended the ramp faster using the C-Leg. Subjects ascended the stairs at a faster pace (not significant) using the PK. This result was most likely due to the study's methodology requiring subjects to ascend the 


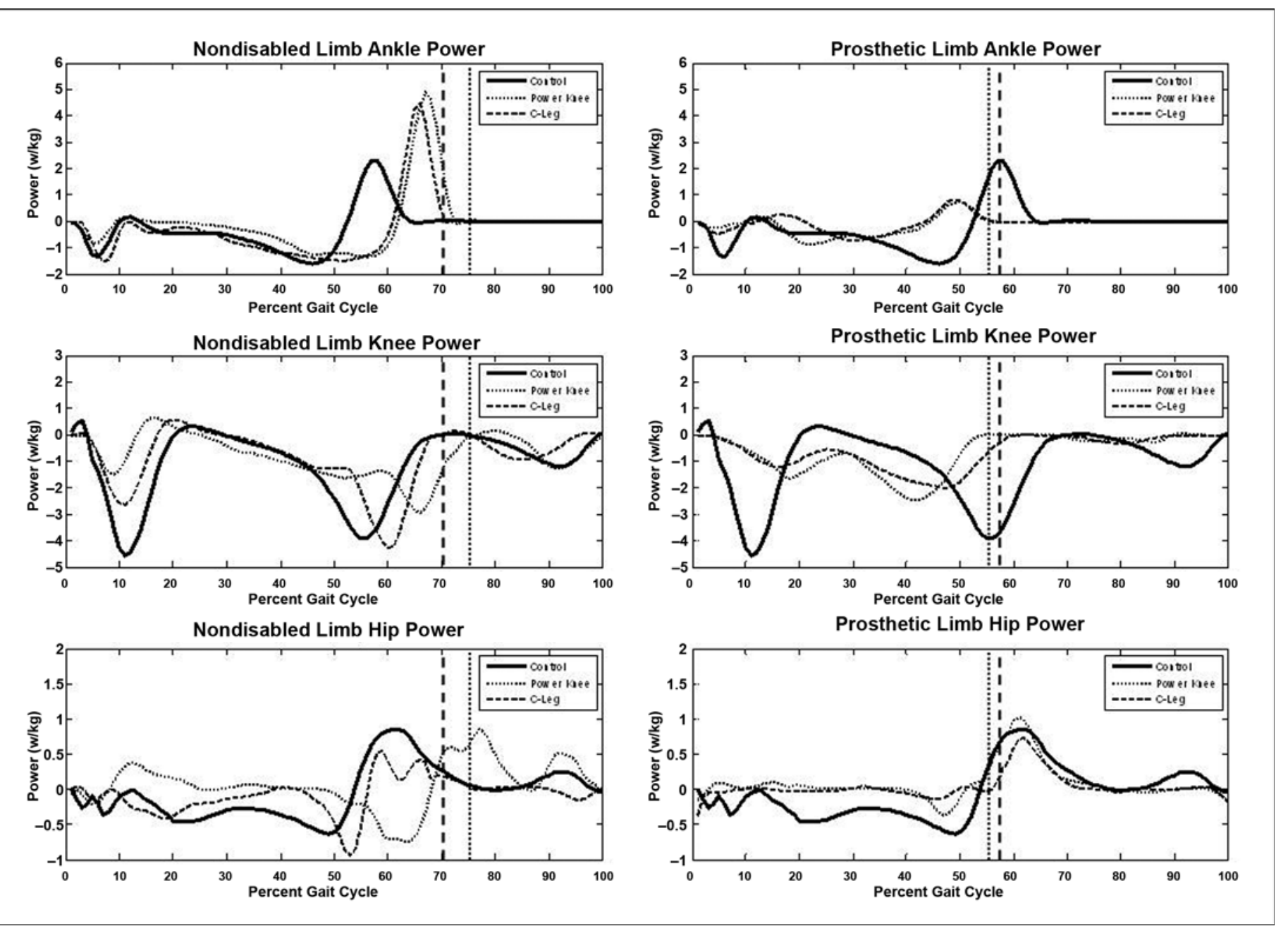

Figure 1.

Ankle, knee, and hip power (W/kg) during ramp descent for servicemembers with transfemoral amputation using C-Leg and Power Knee (representative data from single subject). Foot-off is represented by vertical lines.

stairs step-over-step. The PK is able to assist with the stair climbing task by actively extending the knee. Most subjects chose to perform the step-over-step task in the C-Leg by thrusting themselves up the stairs with their nondisabled limb. Comparable with stair and ramp descent, subjects spent a greater amount of time on their nondisabled limb during ascent, although there were no significant differences between the different components. Unlike the descent observation, subjects took a longer step with their nondisabled limb during ramp ascent compared with ramp descent, and comparing both prostheses, subjects' step length with their prosthetic limb was significantly greater with the C-Leg. The reversal in step lengths is most likely a compensation to reduce loading on the nondisabled limb. If subjects were to take a longer step with their prosthetic limb, they would have to create additional power with the nondisabled limb to ascend the ramp. By taking a shorter step with the prosthetic limb, they are potentially reducing the wear on the nondisabled limb or reducing their energy expenditure by minimizing their overall power output.

Kinetic analysis during ramp and stair ascent showed significant differences for knee power between the knee units. The knee power displayed for servicemembers in the control group and those with TFA while climbing stairs was bimodal (two distinct power generation peaks are displayed) (Figure 4). During stair climbing, a peak knee power occurs at about 20 percent of the gait cycle 


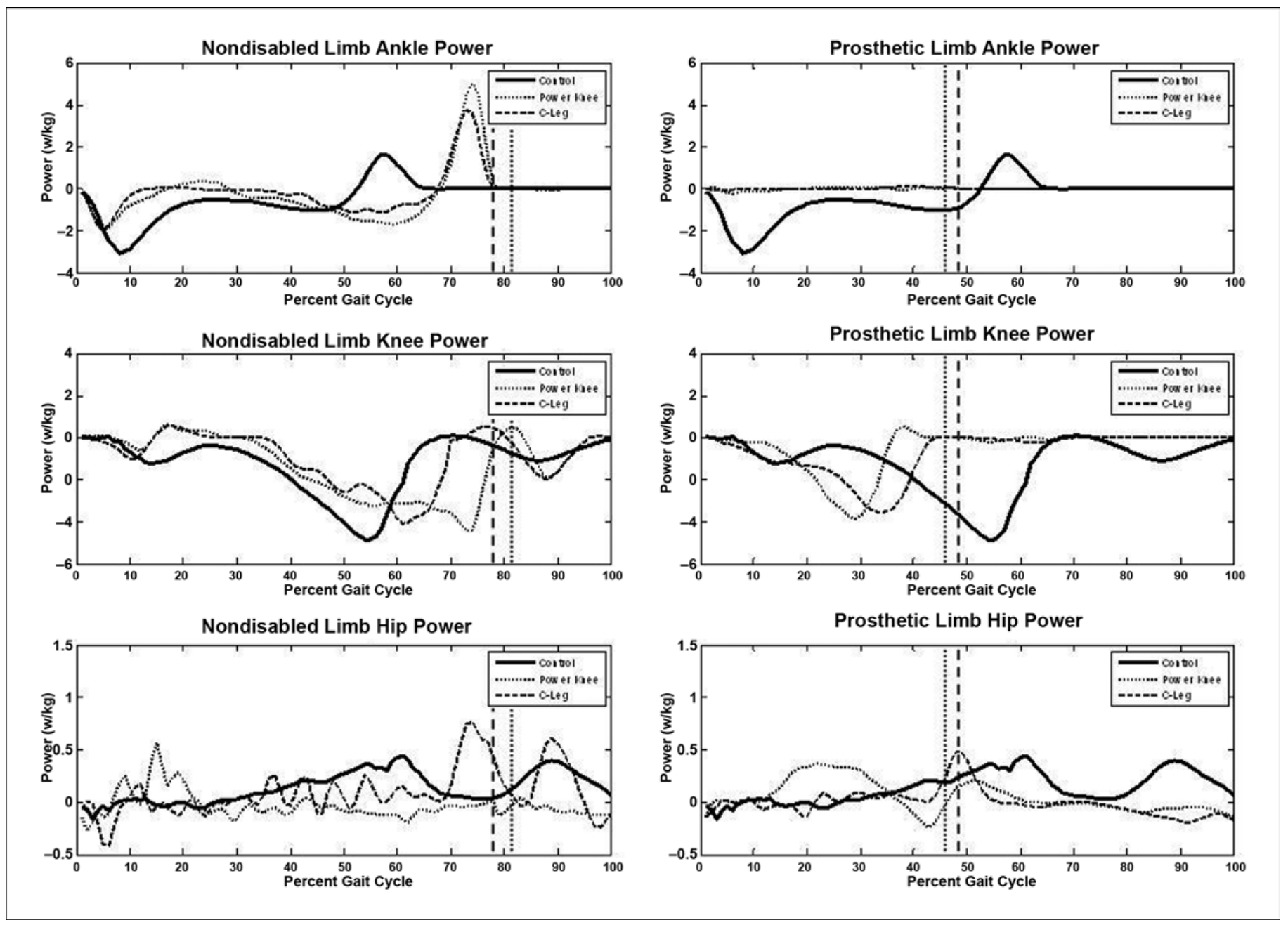

Figure 2.

Ankle, knee, and hip power (W/kg) during stair descent for servicemembers with transfemoral amputation using C-Leg and Power Knee (representative data from single subject). Foot-off is represented by vertical lines.

Table 4.

Temporal-spatial data for ascending ramp and stairs.

\begin{tabular}{|c|c|c|c|c|}
\hline \multirow{2}{*}{ Temporal-Spatial Data } & \multicolumn{2}{|c|}{ Ascending Ramp } & \multicolumn{2}{|c|}{ Ascending Stairs } \\
\hline & Power Knee & C-Leg & Power Knee & C-Leg \\
\hline Nondisabled Stance (\%) & $0.72 \pm 0.06$ & $0.70 \pm 0.04$ & $0.74 \pm 0.03$ & $0.81 \pm 0.07$ \\
\hline Nondisabled Step Length (m) & $0.56 \pm 0.03$ & $0.65 \pm 0.07$ & - & - \\
\hline Prosthetic Step Length (m) & $0.52 \pm 0.09^{*}$ & $0.61 \pm 0.08^{*}$ & - & - \\
\hline
\end{tabular}

*Significant differences between prosthetic components.

after foot strike. This is the power generated by the knee to ascend to the next step. A secondary peak occurs at foot-off. This knee power peak occurs after the contralateral limb has made contact and provides assistance from the trailing limb in ascending to the next step. This secondary peak does not occur during ramp ascension, most likely because the incline is at a level where the ankle of the trailing limb is still providing significant power. 


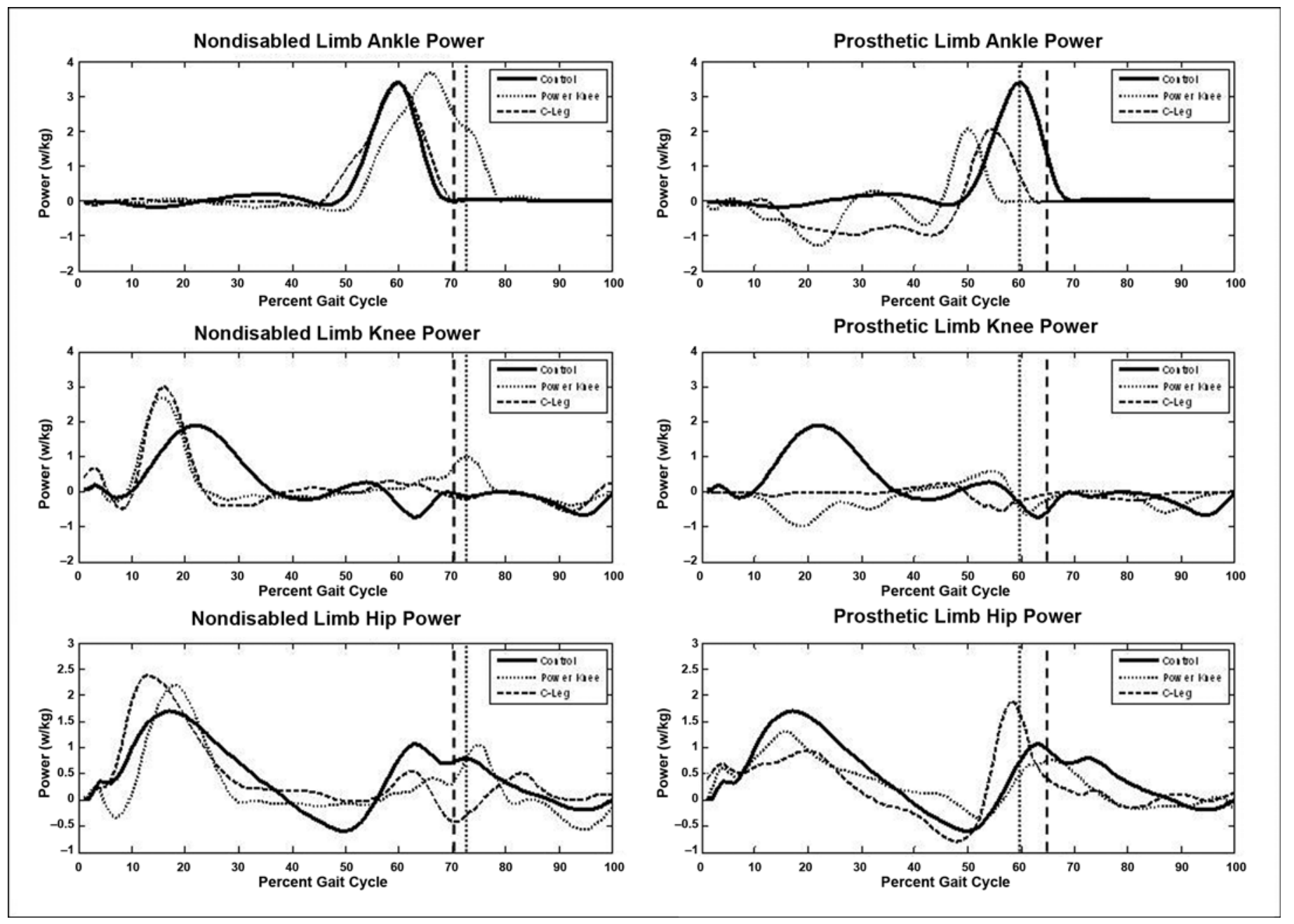

Figure 3.

Ankle, knee, and hip power (W/kg) during ramp ascent for servicemembers with transfemoral amputation using C-Leg and Power Knee (representative data from single subject). Foot-off is represented by vertical lines.

During ramp ascension, the PK does exhibit significantly more peak knee power than the C-Leg. However, the nondisabled limb generated more knee power compared with the pattern displayed by control group servicemembers for both prostheses. The peak knee power generated by the nondisabled limb while using the PK is lower than while using the C-Leg, but it is not statistically significant. For ascending stairs, for the initial peak of the prosthetic limb, the PK generated significantly more power than the C-Leg. This result was expected because of the mechanical design of the PK and the functional limitations of the C-Leg (namely, no active extension). Examination of the secondary peak revealed that, while wearing the C-Leg, the nondisabled limb produced knee power significantly greater than while wearing the PK and the prosthetic limb produced significantly less power. The intact limb of subjects wearing the C-Leg $(9.0 \pm 3.0 \mathrm{~W} / \mathrm{kg})$ exhibited a peak knee power more than four times greater than while wearing the PK $(2.1 \pm 0.3 \mathrm{~W} / \mathrm{kg})$. The prosthetic limb peak knee power generation was four times higher while wearing the PK $(1.2 \pm 0.7 \mathrm{~W} / \mathrm{kg})$ compared with the C-Leg $(0.3 \pm 0.4 \mathrm{~W} / \mathrm{kg})$. This result implies that while ascending stairs step-over-step, subjects with TFA are heaving themselves up with their nondisabled limb while wearing a C-Leg. The total peak knee power generated by the nondisabled limb for the C-Leg $(13.9 \mathrm{~W} / \mathrm{kg})$ while climbing each stair is almost twice that of the PK (7.3 W/kg). 
Table 5.

Power data for ascending ramp and stairs.

\begin{tabular}{|c|c|c|c|c|}
\hline \multirow{2}{*}{ Power Data } & \multicolumn{2}{|c|}{ Ascending Ramp } & \multicolumn{2}{|c|}{ Ascending Stairs } \\
\hline & Power Knee & C-Leg & Power Knee & C-Leg \\
\hline \multicolumn{5}{|c|}{ Peak Ankle Power Generation (W/kg) } \\
\hline Prosthetic & $2.1 \pm 0.5$ & $2.1 \pm 0.5$ & $1.1 \pm 0.5^{*}$ & $0.6 \pm 0.3^{*}$ \\
\hline \multicolumn{5}{|c|}{ Early-Stance Peak Knee Power Absorption (W/kg) } \\
\hline Nondisabled & $2.8 \pm 1.7$ & $3.2 \pm 1.0$ & $5.2 \pm 1.8$ & $4.9 \pm 1.6$ \\
\hline \multicolumn{5}{|c|}{ Late-Stance Peak Knee Power Absorption (W/kg) } \\
\hline Nondisabled & - & - & $2.1 \pm 0.3^{*}$ & $9.0 \pm 3.0^{*}$ \\
\hline Prosthetic & - & - & $1.2 \pm 0.7^{* \dagger}$ & $0.3 \pm 0.4^{* \dagger}$ \\
\hline \multicolumn{5}{|c|}{ Peak Hip Power Generation (W/kg) } \\
\hline Nondisabled & $1.9 \pm 0.8^{*}$ & $2.3 \pm 0.8^{*}$ & $1.9 \pm 0.9$ & $1.7 \pm 0.6$ \\
\hline
\end{tabular}

One of the main limitations of this study is sample size. Only five subjects volunteered to participate in this protocol. This most likely occurred because of the intense training and time commitment required of the subjects. We required subjects to wear both the PK and the C-Leg for 6 weeks and participate in specific training for each knee in addition to their standard physical therapy. Another variable that was unaccounted for was walking speed. We asked subjects to walk at a comfortable speed. Controlling for velocity could have affected the outcomes of the kinetic variables. Subject strength was also not assessed and could be a significant covariate. Variations in strength could be partly controlled by the crossover design of the study and the exclusion of patients with uninjured limb functional deficits. We also asked subjects to perform all of the trials without using handrails and perform the stair conditions step-over-step. These conditions are not necessarily representative of the functional performance of persons with TFA during daily activities, but instead represent an idealistic scenario that prosthetic technologies have not yet achieved. Although not necessarily clinically relevant for most persons with unilateral TFA, performing these tasks in this manner is relevant for this population in regard to their desire to return to Active Duty. It is also noteworthy that since the completion of this study, newer technologies have emerged. The second generation PK (Össur Americas) and the Genium (Otto Bock Healthcare) are now com- mercially available knee units. Both manufacturers claim improvements over former devices.

\section{CONCLUSIONS}

In conclusion, there were functional differences, both temporal-spatial and kinetic, between the PK and the CLeg while ascending and descending ramps and stairs. The main functional differences occurred at the nondisabled and prosthetic knees during stair ascent, a result that was expected because of the design of the PK, which provides active propulsion. The PK was able to significantly reduce the power generated by the nondisabled knee while ascending stairs step-over-step. The C-Leg required users to produce less ankle power generation on the nondisabled limb during stair descent. Also, C-Leg conditions resulted in temporal-spatial differences that included increased speed (although not significant) and greater symmetry between the nondisabled and prosthetic limbs during ramp and stair descent. These data show that significantly more work is required by the nondisabled limb while ascending stairs step-over-step with a prosthesis that does not provide active extension. The data also imply that technology as complex as a powered knee prosthesis may not yet be ideal and only provide a benefit over current knee units during certain tasks. 

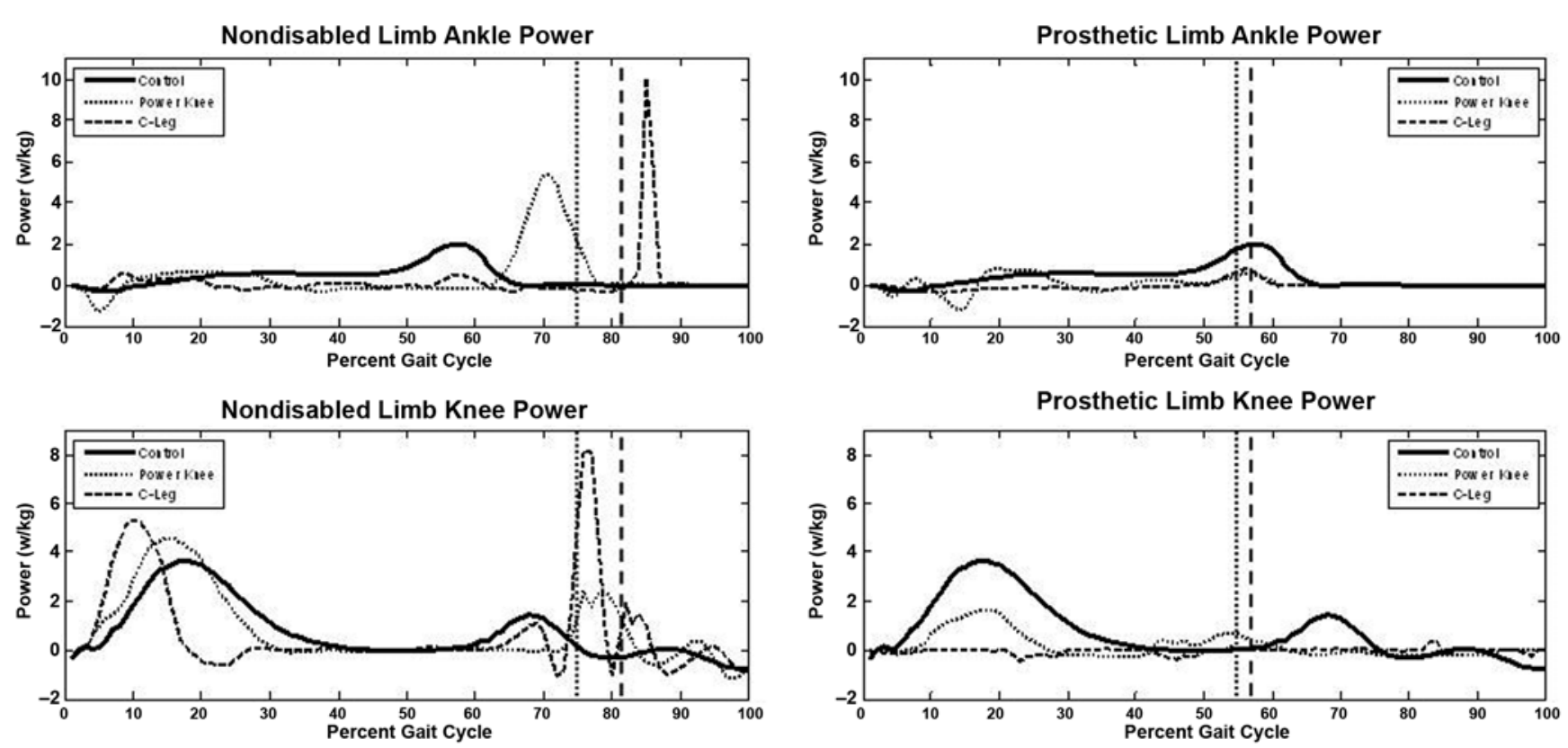

Prosthetic Limb Knee Power
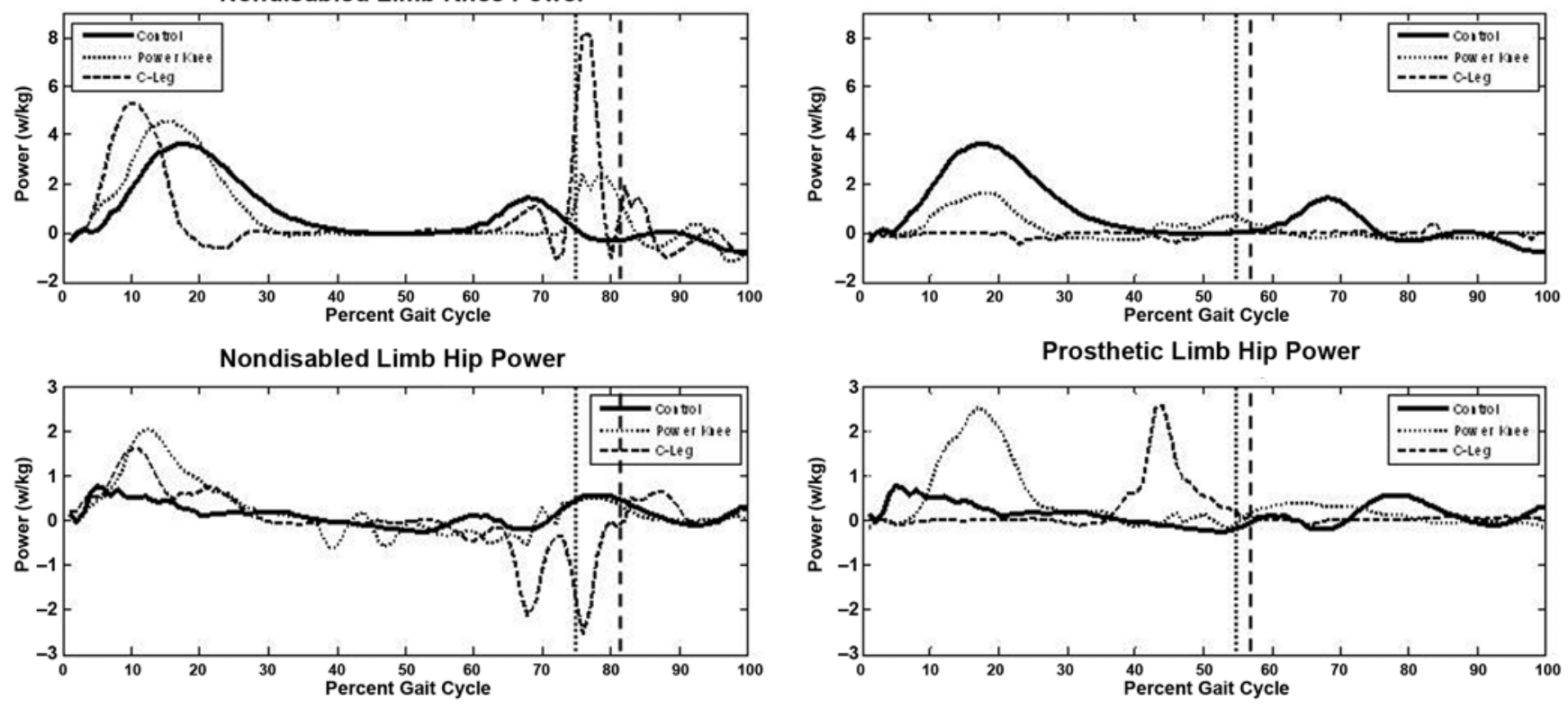

Prosthetic Limb Hip Power

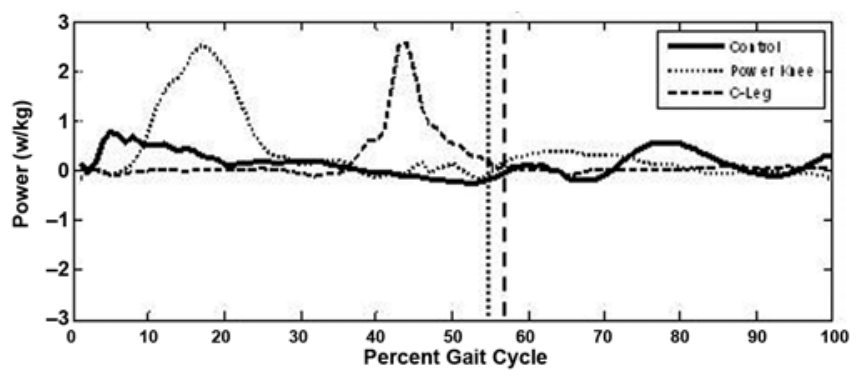

Figure 4.

Ankle, knee, and hip power (W/kg) during stair ascent for servicemembers with transfemoral amputation using C-Leg and Power Knee (representative data from single subject). Foot-off is represented by vertical lines.

\section{ACKNOWLEDGMENTS}

\section{Author Contributions:}

Study concept and design: B. L. Schnall, J. M. Czerniecki, J. M. Gambel.

Acquisition of data: E. J. Wolf, V. Q. Everding, A. L. Linberg, B. L. Schnall.

Analysis and interpretation of data: E. J. Wolf, V. Q. Everding, A. L. Linberg, B. L. Schnall, J. M. Czerniecki, J. M. Gambel.

Drafting of manuscript: E. J. Wolf.

Critical revision of manuscript for important intellectual content: E. J. Wolf, V. Q. Everding, A. L. Linberg, B. L. Schnall,

J. M. Czerniecki, J. M. Gambel.

Statistical analysis: E. J. Wolf.
Obtained funding: B. L. Schnall, J. M. Czerniecki, J. M. Gambel. Study supervision: J. M. Gambel.

Financial Contributions: The authors have declared that no competing interests exist.

Funding/Support: This material was based on work supported by the Military Amputee Research Program.

Institutional Review: The institutional review board of the Department of Clinical Investigations at Walter Reed Army Medical Center approved human subject testing for this protocol.

Additional Contributions: We would like to thank Dave Beachler, Brian Baum, Kathryn Werner, Kat Perret, Paulo Bonato, and Giovani Ortega for their contribution to this work. Drs. Wolf, Linberg, and Gambel; Ms. Everding; and Mrs. Schnall are now with the Department of Orthopedics and Rehabilitation, Walter Reed National Military Medical Center, Bethesda, MD. 
Participant Follow-Up: The authors do not plan to notify participants of the publication of this study because of a lack of contact information.

Disclaimer: The views expressed in this article are those of the authors and do not necessarily reflect the official policy or position of the Department of the Army, Department of Defense, or the U.S. Government.

\section{REFERENCES}

1. Walter Reed Army Medical Center. Amputee database [Internet]. 2009. Available from: https://amputee.wramc.amedd.army.mil

2. Boonstra AM, Schrama J, Fidler V, Eisma WH. The gait of unilateral transfemoral amputees. Scand J Rehabil Med. 1994;26(4):217-23. [PMID:7878397]

3. Chow DH, Holmes AD, Lee CK, Sin SW. The effect of prosthesis alignment on the symmetry of gait in subjects with unilateral transtibial amputation. Prosthet Orthot Int. 2006;30(2):114-28. [PMID:16990222]

http://dx.doi.org/10.1080/03093640600568617

4. Jaegers SM, Arendzen JH, de Jongh HJ. Prosthetic gait of unilateral transfemoral amputees: a kinematic study. Arch Phys Med Rehabil. 1995;76(8):736-43. [PMID:7632129] http://dx.doi.org/10.1016/S0003-9993(95)80528-1

5. Kerrigan DC, Frates EP, Rogan S, Riley PO. Hip hiking and circumduction: quantitative definitions. Am J Phys Med Rehabil. 2000;79(3):247-52. [PMID:10821310] http://dx.doi.org/10.1097/00002060-200005000-00006

6. Miki H, Sugano N, Hagio K, Nishii T, Kawakami H, Kakimoto A, Nakamura N, Yoshikawa H. Recovery of walking speed and symmetrical movement of the pelvis and lower extremity joints after unilateral THA. J Biomech. 2004;37(4):443-55. [PMID:14996556] http://dx.doi.org/10.1016/j.jbiomech.2003.09.009

7. Nolan L, Wit A, Dudziñski K, Lees A, Lake M, Wychowañski M. Adjustments in gait symmetry with walking speed in trans-femoral and trans-tibial amputees. Gait Posture. 2003;17(2):142-51. [PMID:12633775] http://dx.doi.org/10.1016/S0966-6362(02)00066-8

8. Seroussi RE, Gitter A, Czerniecki JM, Weaver K. Mechanical work adaptations of above-knee amputee ambulation. Arch Phys Med Rehabil. 1996;77(11):1209-14. [PMID:8931539] http://dx.doi.org/10.1016/S0003-9993(96)90151-3

9. Burke MJ, Roman V, Wright V. Bone and joint changes in lower limb amputees. Ann Rheum Dis. 1978;37(3):252-54. [PMID:150823] http://dx.doi.org/10.1136/ard.37.3.252

10. Ehde DM, Smith DG, Czerniecki JM, Campbell KM, Malchow DM, Robinson LR. Back pain as a secondary disability in persons with lower limb amputations. Arch Phys
Med Rehabil. 2001;82(6):731-34. [PMID:11387575]

http://dx.doi.org/10.1053/apmr.2001.21962

11. Lemaire ED, Fisher FR. Osteoarthritis and elderly amputee gait. Arch Phys Med Rehabil. 1994;75(10):1094-99. [PMID:7944914] http://dx.doi.org/10.1016/0003-9993(94)90084-1

12. Norvell DC, Czerniecki JM, Reiber GE, Maynard C, Pecoraro JA, Weiss NS. The prevalence of knee pain and symptomatic knee osteoarthritis among veteran traumatic amputees and nonamputees. Arch Phys Med Rehabil. 2005;86(3):487-93. [PMID:15759233] http://dx.doi.org/10.1016/j.apmr.2004.04.034

13. Morgenroth DC, Orendurff MS, Shakir A, Segal A, Shofer J, Czerniecki JM. The relationship between lumbar spine kinematics during gait and low-back pain in transfemoral amputees. Am J Phys Med Rehabil. 2010;89(8):635-43. [PMID:20647781] http://dx.doi.org/10.1097/PHM.0b013e3181e71d90

14. Melzer I, Yekutiel M, Sukenik S. Comparative study of osteoarthritis of the contralateral knee joint of male amputees who do and do not play volleyball. J Rheumatol. 2001;28(1):169-72. [PMID:11196520]

15. Nolan L, Lees A. The functional demands on the intact limb during walking for active trans-femoral and trans-tibial amputees. Prosthet Orthot Int. 2000;24(2):117-25.

[PMID:11061198]

http://dx.doi.org/10.1080/03093640008726534

16. Costigan PA, Deluzio KJ, Wyss UP. Knee and hip kinetics during normal stair climbing. Gait Posture. 2002;16(1):31-37. [PMID:12127184] http://dx.doi.org/10.1016/S0966-6362(01)00201-6

17. Protopapadaki A, Drechsler WI, Cramp MC, Coutts FJ, Scott OM. Hip, knee, ankle kinematics and kinetics during stair ascent and descent in healthy young individuals. Clin Biomech (Bristol, Avon). 2007;22(2):203-10. [PMID:17126461] http://dx.doi.org/10.1016/j.clinbiomech.2006.09.010

18. Schmalz T, Blumentritt S, Marx B. Biomechanical analysis of stair ambulation in lower limb amputees. Gait Posture. 2007;25(2):267-78. [PMID:16725325] http://dx.doi.org/10.1016/j.gaitpost.2006.04.008

19. Narang IC, Mathur BP, Singh P, Jape VS. Functional capabilities of lower limb amputees. Prosthet Orthot Int. 1984;8(1):43-51. [PMID:6718238]

20. Vrieling AH, van Keeken HG, Schoppen T, Otten E, Halbertsma JP, Hof AL, Postema K. Uphill and downhill walking in unilateral lower limb amputees. Gait Posture. 2008;28(2):235-42. [PMID:18242995] http://dx.doi.org/10.1016/j.gaitpost.2007.12.006

21. Buell NC, Willingham LL, Allyn KJ, Hafner BJ, Smith DG, editors. Evaluation of gait style of hill descent for lower limb amputees. 11th World Congress of the International 
Society for Prosthetics and Orthotics; 2004 Aug 1-6; Hong Kong.

22. Chin T, Sawamura S, Shiba R, Oyabu H, Nagakura Y, Takase I, Machida K, Nakagawa A. Effect of an Intelligent Prosthesis (IP) on the walking ability of young transfemoral amputees: comparison of IP users with able-bodied people. Am J Phys Med Rehabil. 2003;82(6):447-51.

[PMID:12820787] http://dx.doi.org/10.1097/01.PHM.0000069191.20125.38

23. Johansson JL, Sherrill DM, Riley PO, Bonato P, Herr H. A clinical comparison of variable-damping and mechanically passive prosthetic knee devices. Am J Phys Med Rehabil. 2005;84(8):563-75. [PMID:16034225]

http://dx.doi.org/10.1097/01.phm.0000174665.74933.0b

24. Hafner BJ, Willingham LL, Buell NC, Allyn KJ, Smith DG. Evaluation of function, performance, and preference as transfemoral amputees transition from mechanical to microprocessor control of the prosthetic knee. Arch Phys Med Rehabil. 2007;88(2):207-17. [PMID:17270519] http://dx.doi.org/10.1016/j.apmr.2006.10.030

25. Kahle JT, Highsmith MJ, Hubbard SL. Comparison of nonmicroprocessor knee mechanism versus C-Leg on Prosthesis Evaluation Questionnaire, stumbles, falls, walking tests, stair descent, and knee preference. J Rehabil Res Dev. 2008;45(1):1-14. [PMID:18566922]

http://dx.doi.org/10.1682/JRRD.2007.04.0054

26. Murray MP, Sepic SB, Gardner GM, Mollinger LA. Gait patterns of above-knee amputee using constant-friction knee components. Bull Prosthet Res. 1980;17(2):35-45.

Submitted for publication December 9, 2010. Accepted in revised form November 16, 2011.

This article and any supplementary material should be cited as follows:

Wolf EJ, Everding VQ, Linberg AL, Schnall BL, Czerniecki JM, Gambel JM. Assessment of transfemoral amputees using C-Leg and Power Knee for ascending and descending inclines and steps. J Rehabil Res Dev. 2012;49(6): 831-42.

http://dx.doi.org/10.1682/JRRD.2010.12.0234

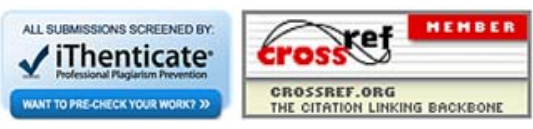

